\title{
Paclitaxel enhances tumoricidal potential of TRAIL via inhibition of MAPK in resistant gastric cancer cells
}

\author{
LIN LI $^{1,2^{*}}$, XIAN-ZI WEN ${ }^{1 *}$, ZHAO-DE BU ${ }^{2}$, XIAO-JING CHENG ${ }^{1}$, XIAO-FANG XING ${ }^{1}$, XIAO-HONG WANG ${ }^{3}$, \\ LIAN-HAI ZHANG ${ }^{2,3}$, TING GUO ${ }^{1}$, HONG DU $^{1}$, YING HU ${ }^{3}$, BIAO FAN ${ }^{2}$ and JIA-FU JI ${ }^{1,2}$ \\ ${ }^{1}$ Key Laboratory of Carcinogenesis and Translational Research (Ministry of Education), Division of Gastrointestinal \\ Cancer Translational Research Laboratory, Peking University Cancer Hospital and Institute, Beijing; Departments of \\ ${ }^{2}$ Gastrointestinal Surgery and ${ }^{3}$ Tissue Bank, Peking University Cancer Hospital and Institute, Beijing, P.R. China
}

Received November 21, 2015; Accepted January 7, 2016

DOI: $10.3892 /$ or.2016.4666

\begin{abstract}
Tumor necrosis factor-related apoptosis-inducing ligand (TRAIL) holds promise for cancer therapy due to its unique capacity to selectively trigger apoptosis in cancer cells. However, TRAIL therapy is greatly hampered by its resistance. A preclinical successful strategy is to identify combination treatments that sensitize resistant cancers to TRAIL. In the present study, we fully assessed TRAIL sensitivity in 9 gastric cancer cell lines. We found combined administration of paclitaxel (PTX) markedly enhanced TRAIL-induced apoptosis in resistant cancer cells both in vitro and in vivo. The sensitization to TRAIL was accompanied by activation of mitochondrial apoptotic pathway, upregulation of TRAIL receptors and downregulation of anti-apoptotic proteins including C-IAP1, C-IAP2, Livin and Mcl-1. Noticeably, we found PTX could suppress the activation of mitogen-activated protein kinases (MAPKs). Inhibition of MAPKs using specific inhibitors (ERK inhibitor U0126, JNK inhibitor SP600125 and P38 inhibitor SB202190) facilitated TRAIL-mediated apoptosis and cytotoxicity. Additionally, SP600125 upregulated TRAL receptors as well as downregulated C-IAP2 and Mcl-1 suggesting the anti-apoptotic role of JNK. Thus, PTX-induced suppression of MAPKs may contribute to restoring TRAIL senstitivity. Collectively, our comprehensive analyses gave new insight into the role of PTX on enhancing TRAIL sensitivity, and provided theoretical references on the development of combination treatment in TRAIL-resistant gastric cancer.
\end{abstract}

Correspondence to: Dr Biao Fan or Dr Jia-Fu Ji, Department of Gastrointestinal Surgery, Peking University Cancer Hospital and Institute, 52 Fu-Cheng Road, Hai-Dian, Beijing 100142, P.R. China E-mail: fanbiao1986@163.com

E-mail: jiafuj@hotmail.com

*Contributed equally

Key words: Tumor necrosis factor-related apoptosis-inducing ligand, paclitaxel, mitogen-activated protein kinase, gastric cancer, apoptosis, combination therapy

\section{Introduction}

Gastric cancer (GC) is the fourth most frequent malignancy and the second leading cause of cancer mortality worldwide (1). In China, most of the patients are identified at the advanced stage which leads to dismal prognosis. Radical surgery is the only curative therapy and chemotherapy serves as common strategy for late stage patients. Patients with GC, however, are not particularly sensitive to conventional chemotherapeutic drugs. Thus, it is of great interest to find new approaches, and targeted manipulation of apoptosis is a research hot spot (2).

TRAIL, a member of tumor necrosis factor (TNF) super family, selectively triggers cell death in transformed cells while causing virtually no toxic towards normal cells (3). TRAIL initiates apoptotic signals via binding to two cell surface death receptors (DRs), DR4 (also known as TRAIL-R1) and DR5 (also known as TRAIL-R2), leading to receptor aggregation and recruitment of Fas-associated death domain (FADD) followed by procaspase- 8 (4). The apoptotic process then follows two signaling pathways. In type I cells, activated caspase- 8 enables the autocatalytic cleavage of caspase cascade triggers apoptosis. While in type II cells, caspase- 8 additionally triggers mitochondrial apoptosis pathway by activating cleavage of Bid, which induces the release of cytochrome $c$ from mitochondria and in turn activates caspase- 9 to finally execute cell death (5). Drugs targeting TRAIL pathway, including recombinant TRAIL and agonistic antibodies have been demonstrated with robust anticancer activity in a number of preclinical studies (6-8).

Despite the attractive tumoricidal potential, TRAILbased therapy is greatly hampered by its resistance, a major obstacle to clinical application (8-10). The most basic cause is dysfunction of death receptors due to hyper-methylation (11), mutation (12) and loss of cell surface expression (13). The defects in caspase protein (14) and overexpression of prosurvival proteins, such as IAP family and anti-apoptotic Bcl-2 family members (15-18), are also linked to TRAIL sensitivity. In addition, more recent findings suggested that cell survival signals, including mitogen-activated protein kinase (MAPK) pathway, phosphatidylinositol 3-kinase/Akt pathway, and transcription factor NF- $\mathrm{B}$ played pivotal roles in regulation of TRAIL signaling (19-22). Previous studies 
demonstrated that TRAIL resistance could be alleviated or even reversed by combination therapy $(23,24)$. PTX, one of the cytoskeletal drugs targeting tubulin, is commonly used in advanced GC treatment. It inhibits tumor cell proliferation through stabilizing microtubule network and inhibiting microtubule dynamics (25). PTX has been explored as sensitizing agent towards TRAIL in some cancer types (26-28), yet, the molecular mechanisms were not fully elucidated.

In the present study, we demonstrate that combined treatment of PTX and TRAIL significantly boosted apoptosis of TRAIL-resistant GC cells both in vitro and in vivo. The molecular mechanisms underlying the synergism involved enhanced activation of caspases, upregulation of DRs and downregulation of anti-apoptotic proteins. Moreover, we present herein the inhibitory effect of MAPKs in TRAILinduced apoptosis in GC and reveal for the first time that TRAIL-augmenting effect of PTX was partly due to suppression of the MAPK pathway.

\section{Materials and methods}

Cell culture and reagents. GC cell lines AGS, NCI-N87, SNU-1 and SNU-16 were purchased from the American Type Culture Collection (ATCC; Manassas, VA, USA). MKN28 and NUGC3 were purchased from Health Science Research Resources Bank (Tokyo, Japan), and SGC-7901, BGC-823 and MGC-803 were obtained from Cell Research Institute (Shanghai, China). All cells were maintained in Dulbecco's modified Eagle's medium (DMEM; Gibco-BRL, Carlsbad, CA, USA) containing 10\% fetal bovine serum (FBS) and penicillin/streptomycin at $37^{\circ} \mathrm{C}$ with $5 \% \mathrm{CO}_{2}$. Recombinant human TRAIL was purchased from PeproTech EC Ltd. (London, UK). The small molecule inhibitors U0126 (\#S1102), SP600125 (\#S1460) and SB202190 (\#S1077) were obtained from Selleck Chemicals (Houston, TX, USA). The chemotherapeutic drug PTX was from Beijing Union Pharmaceutical Factory (Beijing, China).

Cell viability assay. Cell viability was assessed using Cell Counting kit-8 assay (CCK-8) (Dojindo Molecular Technologies, Kumamoto, Japan). Briefly, cells were seeded onto 96-well plates at a density of $3-5 \times 10^{3}$ cells/well and allowed to attach for $24 \mathrm{~h}$. The cells were incubated with indicated concentrations of TRAIL and/or PTX for $24 \mathrm{~h}$. After incubation, CCK-8 solution $(10 \mu \mathrm{l})$ was added to each well and absorbance was measured at $450 \mathrm{~nm}$ using multiscan spectrum.

Flow cytometry for apoptosis analysis and expression of cell surface DRs. For cell apoptosis assay, cells $\left(3 \times 10^{5}\right)$ were seeded onto $35-\mathrm{mm}^{2}$ culture dishes and treated with TRAIL and/or PTX for $24 \mathrm{~h}$ as indicated in the figure legends. Then, the cells were stained using an Annexin V/PI double staining kit (Dojindo Molecular Technologies) according to the manufacturer's protocol.

For cell surface expression analysis, cells $\left(1 \times 10^{6}\right)$ treated with PTX or vehicle for $24 \mathrm{~h}$ were incubated with allophycocyanin (APC)-conjugated anti-DR4 and anti-DR5 antibody, or isotype control (BioLegend, Inc., San Diego, CA, USA) for 30 min at $4^{\circ} \mathrm{C}$, and analyzed by flow cytometery (BD Accuri C6; BD Biosciences, San Jose, CA, USA). Positive cells were identified and measured by subtracting relative isotype control values.

Western blot assay. Total proteins were extracted using RIPA lysis buffer (Beijing Solarbio Science and Technology Co., Ltd., Beijing, China) with protease inhibitors and phosphatase inhibitors (Beijing Solarbio Science and Technology). Protein concentration was quantified using the Bradford method (Pierce, Rockford, IL, USA). Equivalent amount of protein $(20 \mu \mathrm{g})$ was separated by $10 \%$ SDS-PAGE gels and transferred onto PVDF membranes (Millipore, Billerica, MA, USA). The membranes were blocked in 5\% non-fat milk in TBS-T for $1 \mathrm{~h}$ and incubated with primary antibodies overnight at $4{ }^{\circ} \mathrm{C}$, followed by incubation with HRP-conjugated secondary antibodies. Signals were detected using chemiluminescent agents (Pierce). Primary antibodies of DR4 and GAPDH were from Abcam (Cambridge, MA, USA), and DR5 was from SigmaAldrich (St. Louis, MO, USA). Other primary antibodies purchased from Cell Signaling Technology (Danvers, MA, USA) were as follows: caspase-3 (\#9662), cleaved-caspase-3 (\#9664), caspase-7 (\#9492), cleaved-caspase-7 (\#8438), caspase-8 (\#9746), caspase-9 (\#9502), cleaved-caspase-9 (\#7237), PARP (\#9542), cleaved-PARP (\#5625), Bid (\#2002), C-IAP1 (\#7065), C-IAP2 (\#3130), XIAP (\#2045), Livin (\#5471), Bcl-xL (\#2764), Bcl-2 (\#2870), Mcl-1 (\#5453), ERK (\#4695), phosphorylated-ERK (\#4370), JNK (\#9258), phosphorylatedJNK (\#4668), p38 (\#8690), phosphorylated-p38 (\#4511).

siRNA transfection. C-IAP1 siRNA and scrambled siRNA were purchased from Santa Cruz Biotechnology (Santa Cruz, CA, USA). Cells $\left(1 \times 10^{5}\right)$ in 6 -well plates were incubated for $24 \mathrm{~h}$ and then transfected with siRNA (100 nmol/l) and Lipofectamine 2000 reagent (Invitrogen, Carlsbad, CA, USA) in Opti-MEM without serum, according to the manufacturer's specifications. After $6 \mathrm{~h}$, the medium was replaced with fresh medium. Forty-eight hours after transfection, C-IAP1 knockdown effect was verified by western blotting.

In vivo xenograft study. Animal studies were carried out in strict adherence with institutional guidelines. SGC-7901 cells (2x106/200 $\mu \mathrm{l}$ per mouse) were subcutaneously injected into the right hind legs of 6-8 week-old female nude mice. When tumors volume reached $50-100 \mathrm{~mm}^{3}$, the mice were randomized to 4 groups and dosing was initiated. They were: i) control (vehicle only); ii) TRAIL (100 $\mu \mathrm{g} / \mathrm{kg}$ intratumoral injection); iii) PTX (10 mg/kg i.p.); and iv) the sequential administration of PTX (10 mg/kg i.p.) followed by TRAIL (100 $\mu \mathrm{g} / \mathrm{kg}$ intratumoral injection) after $24 \mathrm{~h}$. All groups were treated once every 3 days for 18 days. The tumor size and weight were monitored three times a week. Tumor volume (V) was calculated as $\mathrm{V}=0.5 \mathrm{x}$ length $\mathrm{x}$ width $^{2}$. Tumor growth inhibition (TGI) was assessed in accordance with the formula $\left[1-\left(\mathrm{T}-\mathrm{T}_{0}\right) /\left(\mathrm{C}-\mathrm{C}_{0}\right)\right] \mathrm{x}$ 100 , where $\mathrm{T}$ and $\mathrm{T}_{0}$ were the mean tumor volumes at the end of the drug administration and day 0 , respectively for treated group, and $\mathrm{C}-\mathrm{C}_{0}$ were those for vehicle control group.

Statistical analysis. All the values are presented as mean \pm SD of three independent experiments. The statistical significance among experimental groups was assessed by two-sided Student's t-test. $\mathrm{P}<0.05$ were considered to be significant. All 
A
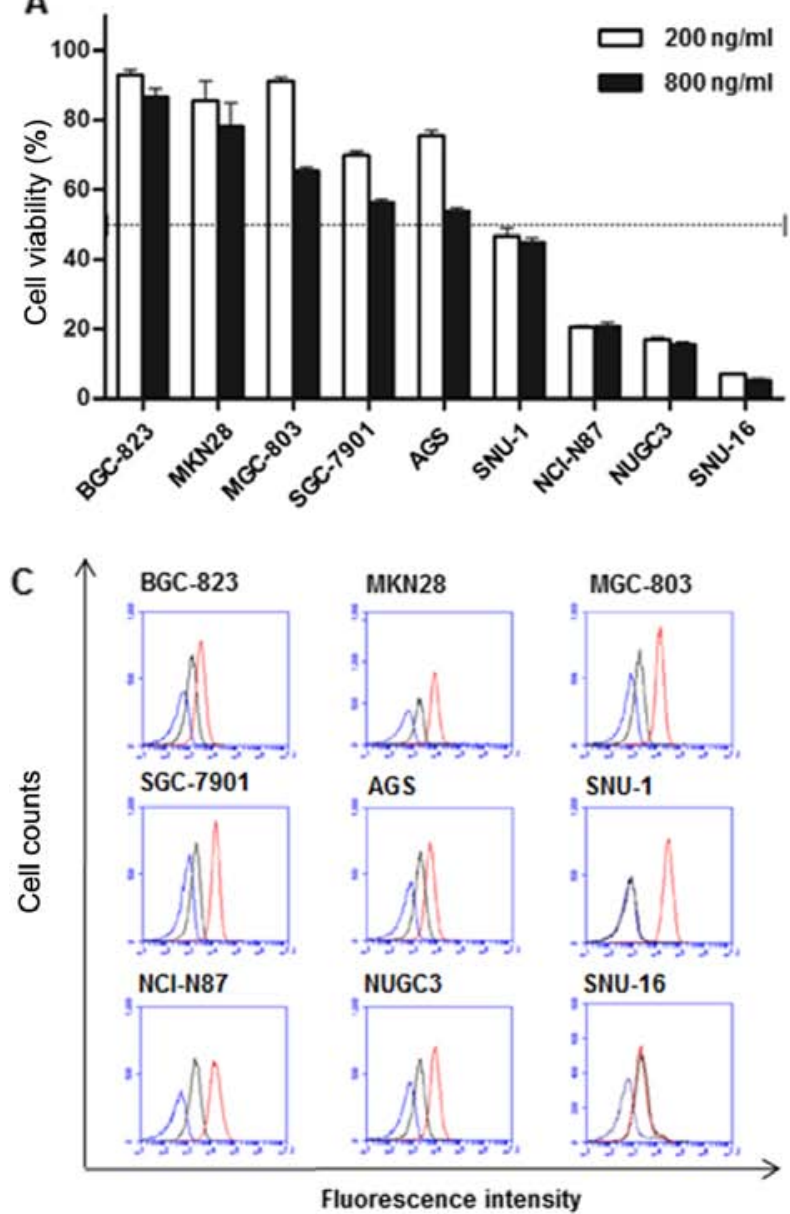

B
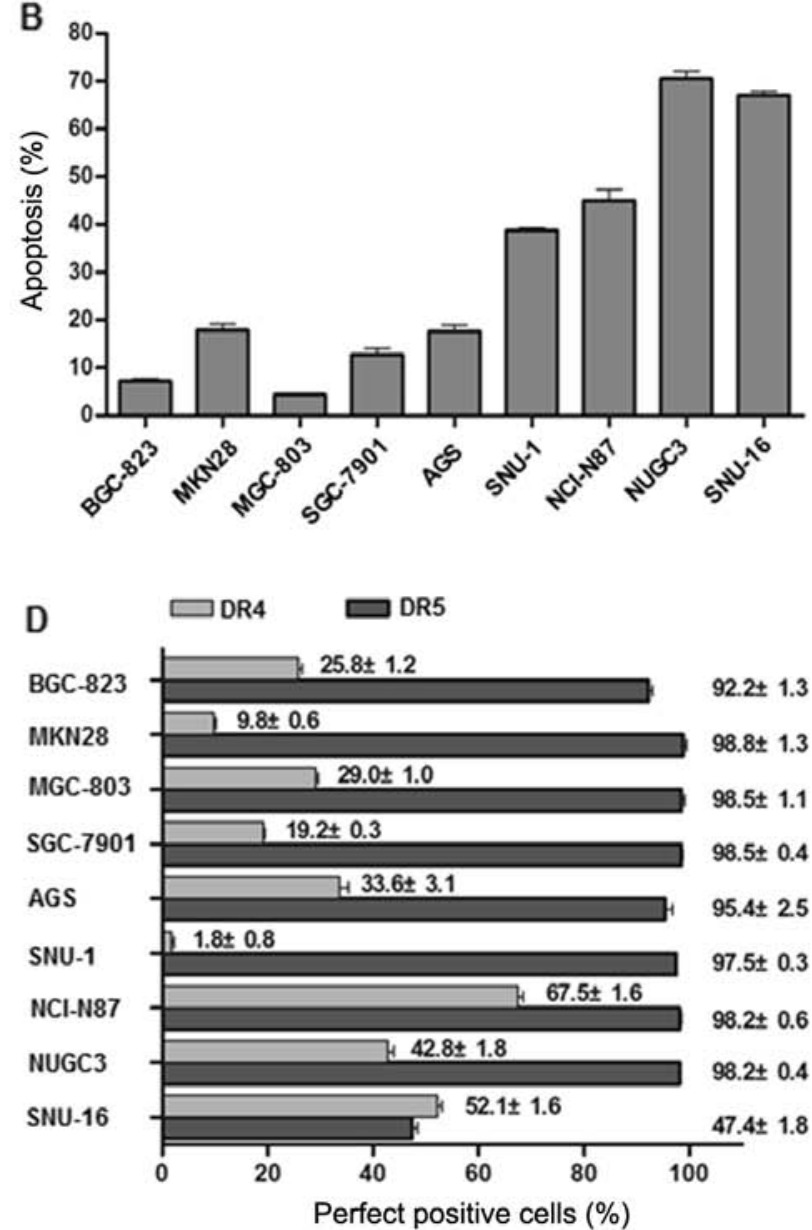

Figure 1. The sensitivity of GC cells to TRAIL and expression of death receptors. (A) CCK-8 assay for 9 GC cell lines after incubation with TRAIL at indicated doses for $24 \mathrm{~h}$. (B) Apoptotic analysis for $9 \mathrm{GC}$ cell lines after incubation with TRAIL (200 ng/ml) for $24 \mathrm{~h}$. (C) Cell surface expression of DR4 and DR5 assessed by flow cytometric method. Blue, black and red lines indicate isotype control, DR4 and DR5, respectively. (D) Quantified DR4 and DR5 expression on cell surface.

statistical analyses were performed using GraphPad Prism 5.0 software.

\section{Results}

Response of GC cells to TRAIL and expression of TRAIL receptors. First, we analyzed the effects of TRAIL on cell viability in 9 GC cells using CCK-8 assay. As shown in Fig. 1A, GC cells had varying degree of sensitivity to TRAIL. SNU-16, NUGC3, NCI-N87 and SNU-1 were defined as sensitive cells, in which $>50 \%$ growth inhibition was achieved when treated with TRAIL (200 ng/ml) for $24 \mathrm{~h}$. By contrast, the growth inhibition rates of TRAIL-resistant cells (BGC-823, MKN28, MGC-803, SGC-7901 and AGS) were $<50 \%$ even under condition of elevated concentration or prolonged exposure time indicating an intrinsic resistance mechanisms in these cells. TRAIL-induced apoptosis was also confirmed by Annexin V/PI double staining in the same cell lines (Fig. 1B). To determine whether TRAIL pathway was intact, we measured cell surface expression of DR4 and DR5 (Fig. 1C and D). Flow cytometric analysis revealed a predominant expression of DR5 in all these cells except SNU-16. DR4, however, exhibited diverse levels in 9 cell lines and the top three sensitive cells (SNU-16, NUGC3 and NCI-N87) expressed the highest levels.
PTX and TRAIL act synergistically to inhibit GC cell growth. PTX exhibited growth inhibiting effect in TRAIL-resistant cell lines (Fig. 2A). We attempted to investigate whether PTX augments TRAIL-inducing apoptosis and found a significant decrease on cell viability in combination group compared with TRAIL or PTX alone in all 5 resistant cell lines $(\mathrm{P}<0.05)$ (Fig. 2B). To confirm this synergism, SGC-7901 and MGC-803 cells were cultured with different combinations of TRAIL (100 and $200 \mathrm{ng} / \mathrm{ml}$ ) and PTX (5 and $10 \mathrm{nM})$. The results indicated that PTX promoted TRAIL-mediated cytotoxic effect in a dose-dependent manner (Fig. 2C). Considering changes in the order of drug exposure could enhance cell death (29) and sequential application of PTX followed by other anticancer chemicals is common in GC treatment (30), we tested the possibility of sequential administration of PTX and TRAIL. Cells were pre-incubated with PTX alone for $12 \mathrm{~h}$ and subsequently treated with TRAIL for an additional $12 \mathrm{~h}$. Reduction of cell viability was more distinct in the sequential exposure group compared with simultaneous administration group (Fig. 2D), indicating that pre-treatment of PTX rendered cancer cell more vulnerable to TRAIL.

PTX sensitizes GC cells to TRAIL-induced apoptosis by activating caspase-dependent mitochondrial apoptotic pathway. 

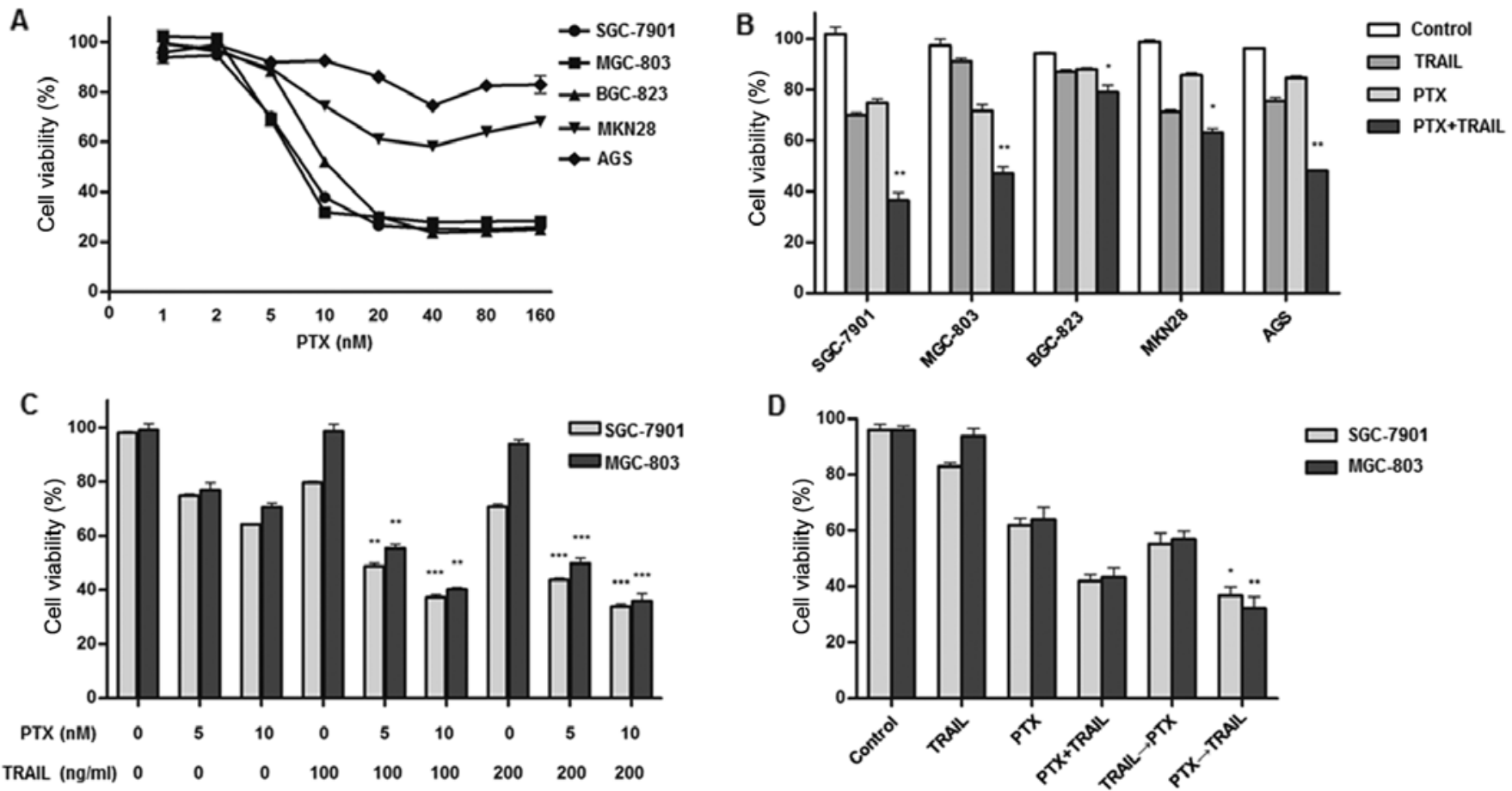

Figure 2. PTX potentiated TRAIL-induced cytotoxicity in GC cells. (A) CCK-8 assay for five TRAIL-resistant cell lines after incubation with PTX at indicated doses for $48 \mathrm{~h}$. (B) CCK-8 assay for five TRAIL-resistant cell lines after 24-h incubation with $5 \mathrm{nM} \mathrm{PTX,} 200 \mathrm{ng} / \mathrm{ml}$ TRAIL or the combination of TRAIL and PTX, respectively. (C) CCK-8 assay for SGC-7901 and MGC-803 cells after 24-h exposure to TRAIL and/or PTX at indicated doses. (D) Effect of sequential application of PTX and TRAIL on cell viability. SGC-7901 and MGC-803 cells were treated with PTX (10 nM) and TRAIL (100 ng/ml) for $24 \mathrm{~h}$, or pretreated with PTX for $12 \mathrm{~h}$ followed by TRAIL for another $12 \mathrm{~h}$, or treated in reverse order of the former, respectively. ${ }^{*} \mathrm{P}<0.05,{ }^{* *} \mathrm{P}<0.01,{ }^{* * * *} \mathrm{P}<0.001$.
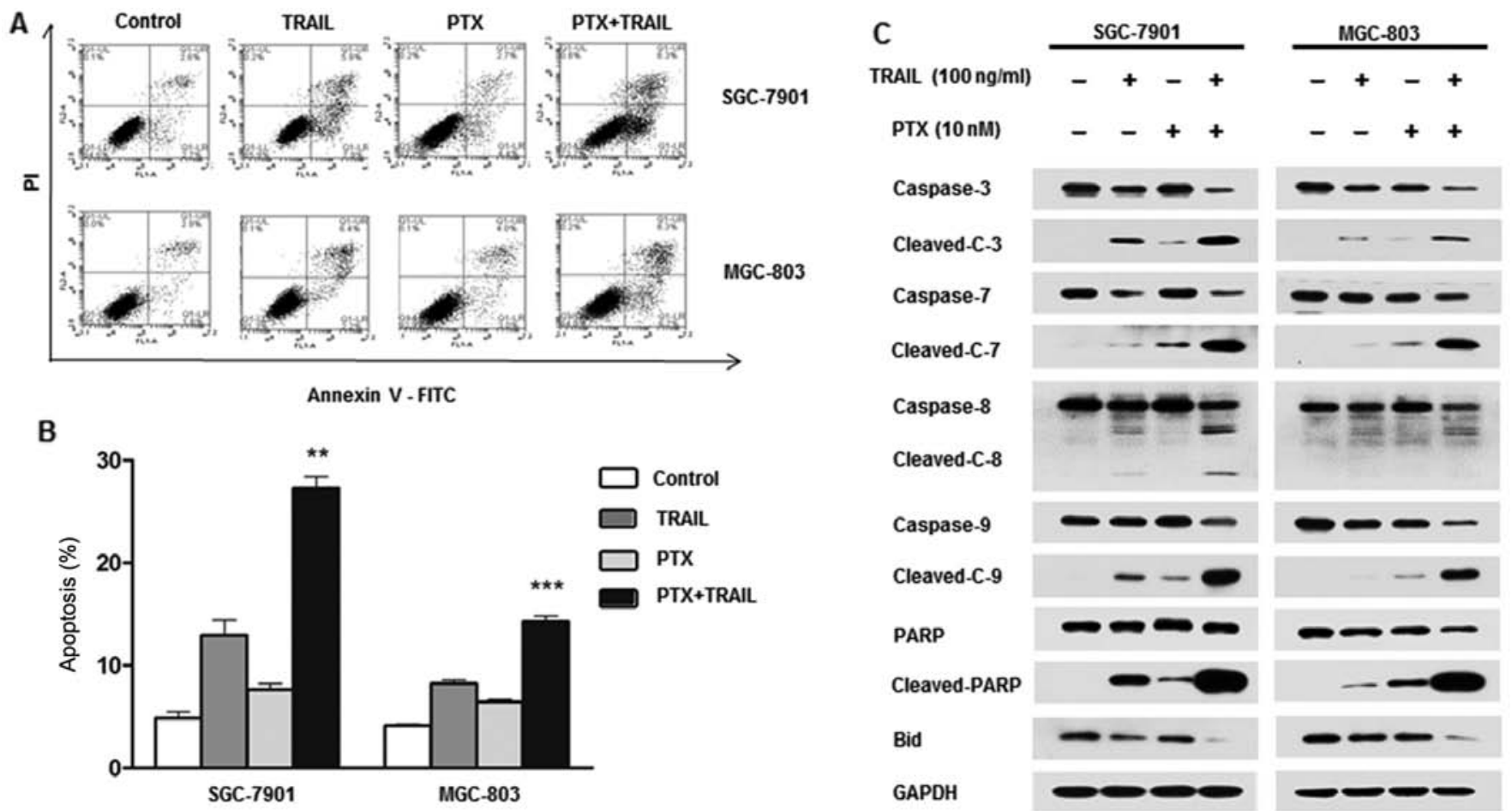

Figure 3. PTX sensitizes TRAIL-induced apoptosis by activating the caspase-dependent pathway. (A and B) Apoptosis rate was assessed by Annexin V/PI staining in SGC-7901 and MGC-803 cells after 24-h treatment with TRAIL (100 ng/ml) and/or PTX (10 nM). (C) Caspase cleavage was determined by western blotting after SGC-7901 and MGC-803 cells were treated with TRAIL $(100 \mathrm{ng} / \mathrm{ml})$ and/or PTX (10 nM) for $16 \mathrm{~h} .{ }^{* *} \mathrm{P}<0.01,{ }^{* * * *} \mathrm{P}<0.001$.

To ascertan whether cell growth inhibition was due to apoptosis, Annexin V/PI double staining was performed. A significant increase in apoptosis was observed in the combination treatment group compared with either agent alone 

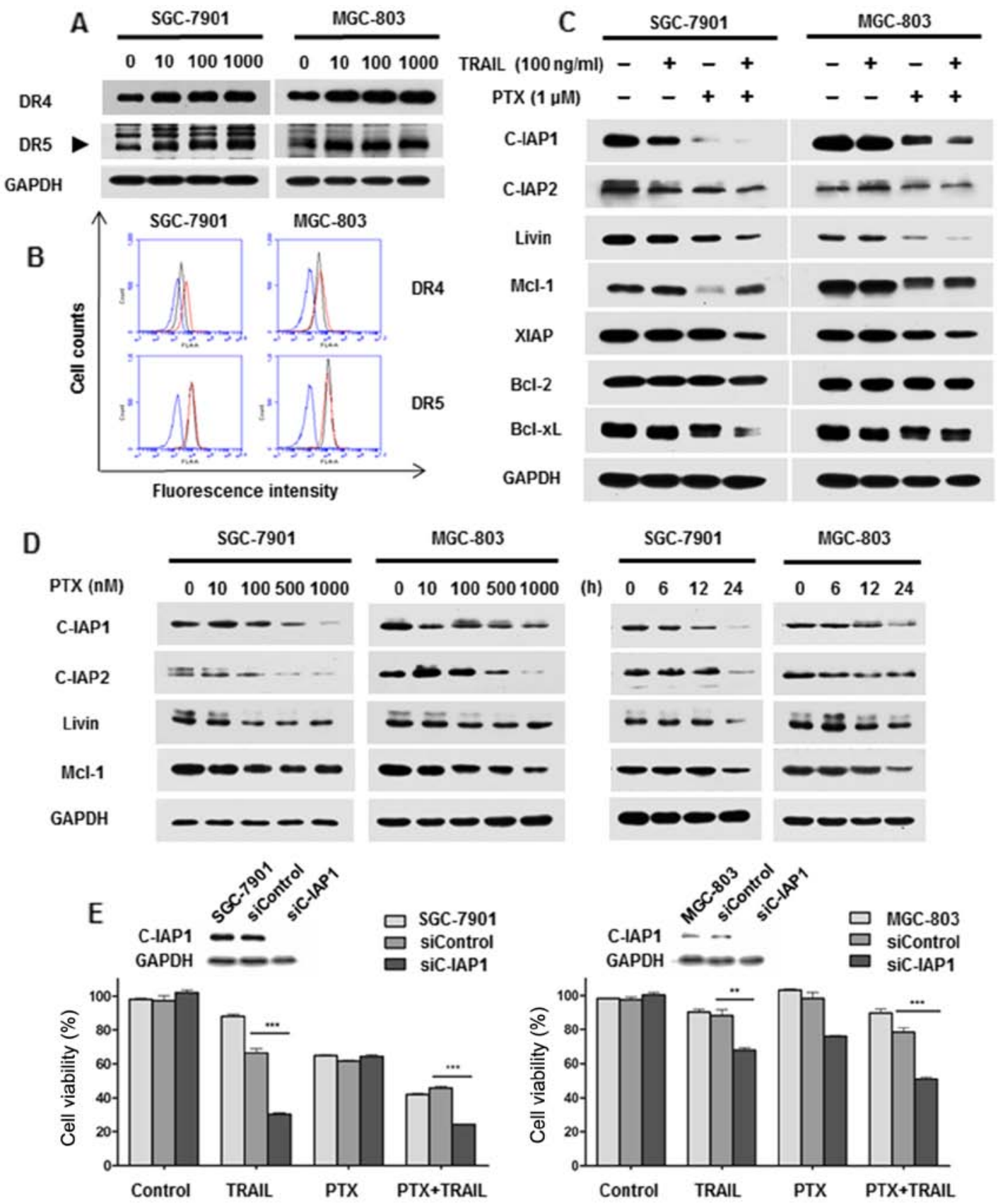

Figure 4. PTX altered expression of death receptors and anti-apoptotic proteins. (A) DR4 and DR5 expression was assessed by western blotting in SGC-7901 and MGC-803 cells after 24-h incubation with PTX at indicated doses. (B) Cell surface expression of DR4 and DR5 was assessed by flow cytometric method in SGC-7901 and MGC-803 cells after incubation with $100 \mathrm{nM}$ PTX for $24 \mathrm{~h}$. The blue lines indicate staining with isotype control, black lines and red lines indicate absence and presence of PTX, respectively. (C) SGC-7901 and MGC-803 cells were treated with TRAIL (100 ng/ml) and/or PTX (1 $\mu \mathrm{M})$ for $24 \mathrm{~h}$. Anti-apoptotic proteins were analyzed by western blotting. (D) SGC-7901 and MGC-803 cells were treated with indicated doses of PTX for $24 \mathrm{~h}$ or $1 \mu \mathrm{M}$ PTX for indicated time-points. Anti-apoptotic proteins were analyzed by western blotting. (E) Effect of C-IAP1 silencing on cell viability induced by TRAIL plus PTX. SGC-7901 and MGC-803 cells were transiently transfected with C-IAP1 specific siRNA for $48 \mathrm{~h}$ and then incubated with TRAIL (100 ng/ml) and/or PTX $(10 \mathrm{nM})$ for $24 \mathrm{~h}$. Cell viability was analyzed by the CCK-8 assay. ${ }^{* *} \mathrm{P}<0.05,{ }^{* * *} \mathrm{P}<0.01$.

(Fig. 3A and B). Taking SGC-7901 cell line as an example, the mean apoptotic rate was 2.8 and $8 \%$ treated with PTX and TRAIL alone, respectively, but as much as $22.4 \%$ after combination treatment. The expression of full length and cleaved caspase-3, $-7,-8$ and -9 and PARP was measured by western blotting (Fig. 3C). Combination of TRAIL and PTX markedly enhanced cleavage of all the caspase proteins compared with single agent alone. Noteworthy, cleavage of caspase-9, but not caspase-8, was induced by PTX, indicating the effect of PTX on activation of mitochondrial pathway. We further tested expression of Bid, another key component in mitochondrial pathway, which was significantly decreased in combination group.

PTX upregulated TRAIL receptors and downregulated antiapoptotic proteins. Given the therapeutic potential of combined regimen, we focused the following studies on unraveling the molecular mechanism of the synergism. As shown in Fig. 4A, PTX induced DR4 and DR5 expression in both SGC-7901 and MGC- 803 cells, albeit the trend was not distinct in MGC-803 

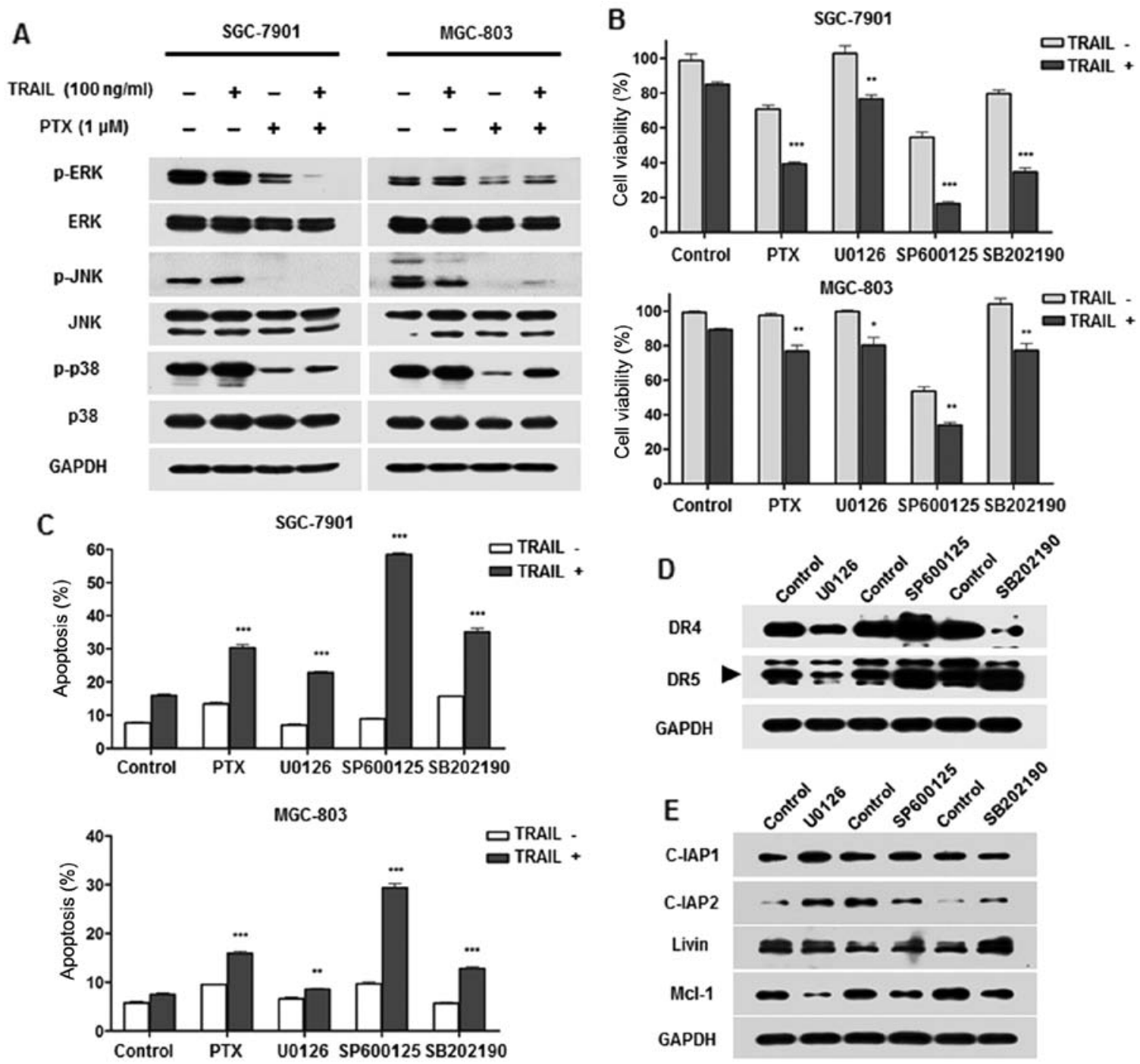

Figure 5. MAPK pathway was involved in PTX-mediated sensitization to TRAIL. (A) Western blotting assay showed alteration of p-ERK, p-JNK and p-p38 in SGC-7901 and MGC-803 cells after exposure to TRAIL $(100 \mathrm{ng} / \mathrm{ml})$ and/or PTX $(1 \mu \mathrm{M})$ for $24 \mathrm{~h}$. (B) CCK-8 assay and (C) Annexin V/PI staining assay for SGC-7901 and MGC-803 cells treated with TRAIL (100 ng/ml) in the absence or presence of PTX $(1 \mu \mathrm{M}), \mathrm{U} 0126(10 \mu \mathrm{M}), \mathrm{SP} 600125(10 \mu \mathrm{M})$ and SB202190 $(10 \mu \mathrm{M})$, respectively. ${ }^{*} \mathrm{P}<0.05,{ }^{* *} \mathrm{P}<0.05,{ }^{* * * *} \mathrm{P}<0.01$. (D) Expression of DRs and (E) anti-apoptotic proteins was assessed by western blotting after SGC-7901 cells were treated with $\mathrm{U} 0126(10 \mu \mathrm{M})$, SP600125 $(10 \mu \mathrm{M})$ or SB202190 $(10 \mu \mathrm{M})$, respectively.

cells. Additionally, enhanced cell surface expression of DR4, not DR5, was also examined after PTX treatment by flow cytometric analysis (Fig. 4B). To further uncover the role of PTX on modulating cell death, apoptosis-related proteins were investigated. Notably, combination treatment significantly inhibited expression of anti-apoptotic regulators including C-IAP1, C-IAP2, Livin and Mcl-1 (Fig. 4C). Consistent with these findings, decreased expression of these proteins was also observed in SGC-7901 and MGC-803 cells treated with PTX alone in a dose- and time-dependent manner (Fig. 4D). Moreover, a representative protein C-IAP1 was chosen for functional verification. Silencing of C-IAP1 by siRNA enhanced TRAIL-mediated cytotoxicity and amplified the TRAIL-sensitizing effect of PTX (Fig. 4E). All these results suggested that PTX sensitized TRAIL-induced apoptosis via upregulating DRs and downregulating anti-apoptotic proteins.

PTX induced inactivation of the MAPK pathway. Considering MAPKs have been implicated in regulation of TRAIL- resistance $(19,31)$ in conjunction with the reports that PTX could modulate activation of MAPKs (32), we hypothesized that PTX-mediated MAPK activity may contribute to TRAIL sensitivity in GC cells. To this end, we first evaluated the effect of PTX on MAPK activity. Results depicted in Fig. 5A demonstrated ERK, JNK and p38 were strongly suppressed after treatment of PTX alone as well as in combination with TRAIL. Next, we investigated the possible role of MAPK deprivation on the sensitivity to TRAIL. Treatment of U0126 (ERK inhibitor), SP600125 (JNK inhibitor) and SB202190 (p38 inhibitor) respectively, significantly enhanced TRAILinduced apoptosis and cytotoxicity, as did PTX (Fig. 5B and C). Among the three inhibitors, SP600125 showed the strongest sensitization effect. To further assess the effect of MAPKs on TRAIL signalling, expression of DRs and anti-apoptotic proteins were evaluated after pretreatment with specific inhibitors. Results showed SP600125 increased DR4 and DR5 expression while U0126 suppressed them indicating MAPKs exhibited opposite effect on DRs (Fig. 5D). Moreover, C-IAP2 


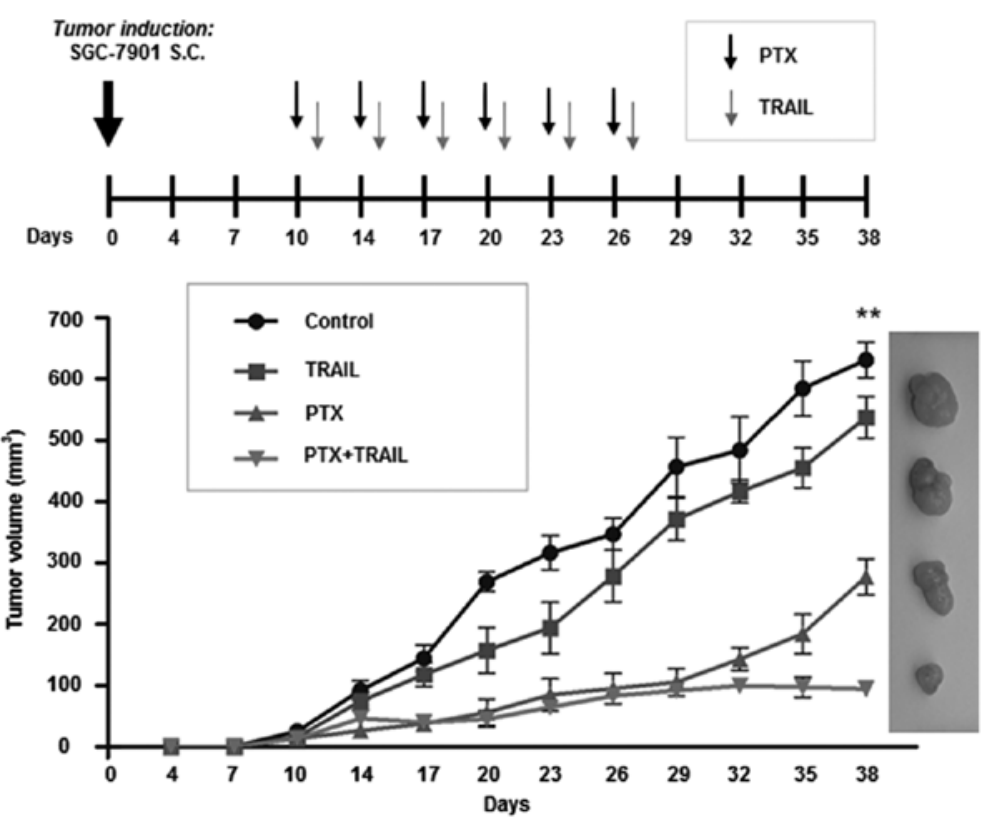

Figure 6. Combination of PTX and TRAIL inhibited tumor growth in the SGC-7901-derived xenograft model. The mice bearing SGC-7901 xenografts were randomly divided into four groups and treated with PBS, TRAIL, PTX or combination once every 3 days for a period of 18 days and tumor volume was measured every 3 days. ${ }^{* *} \mathrm{P}<0.05$.

was suppressed by SP600125 and Mcl-1 was decreased by all three inhibitors (Fig. 5E). Collectively, these results suggested that PTX-mediated inhibition of MAPKs might contribute to facilitate TRAIL potential in GC cells.

PTX enhances the antitumor effect of TRAIL in a tumor xenograft model. To assess the therapeutic effect of PTX and TRAIL, we established nude mouse models bearing SGC-7901 tumor xenografts. The results revealed that TRAIL or PTX alone only slightly suppressed growth of tumors (TGI was 14.8 and $56.0 \%$, respectively), whereas in the combination group, TGI was $85.1 \%$ at the end of drug administration (Fig. 6). The data further confirmed that PTX enhanced the tumor-suppressing capacity of TRAIL in resistant GC cells.

\section{Discussion}

Identification of new drugs causing tumor specific apoptosis has roused enormous interest. TRAIL legend and its receptors are attractive targets for the selective eradication of tumor cells. Recombinant TRAIL protein and TRAIL receptor agonistic antibodies have been tested in clinical trials, displaying encouraging antitumor activities with mild sideeffects. Nevertheless, resistance to TRAIL limits their clinical application. In the present study, we found not all the cell lines underwent significant apoptosis when treated with TRAIL. Numerous chemotherapeutic agents can sensitize tumor cells to TRAIL-mediated apoptosis (26-28). However, there are scarce data elucidating the synergistic interaction between PTX and TRAIL in GC cells. In the present study, we assessed the tumoricidal potential of TRAIL combined with PTX both in vitro and in vivo, and analyzed the mechanism by which PTX sensitized TRAIL-resistant GC cells.

One possible mechanism is synergistic activation of caspase. Apoptosis can be induced by two pathways. TRAIL and PTX share two complementary characteristics for performing apoptosis: one is high efficacy of cell death triggered by death receptor-mediated pathway, and the other is activation of mitochondria-controlled signaling. In the present study, we observed that TRAIL alone only slightly activated caspase-3, -8 and PARP in SGC-7901 and MGC-803 cells. In contrast, simultaneous administration of PTX and TRAIL resulted in a dramatic increase in cleavage of all caspase proteins. Notably, the combined administration markedly activated caspase- 9 and induced cleavage of Bid, which are key events in mitochondrial apoptosis signaling, suggesting that PTX facilitated sensitivity to TRAIL by reinforcing mitochondria-mediated apoptosis. This observation is supported by others reporting that PTX induces apoptosis via a death receptor-independent, caspase-3/-8-driven mitochondrial amplification loop (33).

Apoptosis signals are initiated when TRAIL binds to DR4 and DR5. Upregulation of DRs can enhance the responsiveness of cancer cells to TRAIL-mediated cell death $(26,34)$. According to our data, high DR5 expression was prevalent and almost undifferentiated in GC cells. Intriguingly, GC cells shared diverse surface expression of DR4 with the tendency that TRAIL-resistant cell lines exhibited relatively low levels. These findings provided novel evidence that susceptibility of GC cells to TRAIL might be mainly ascribed to the surface expression of DR4. As we found PTX could increase DR4 expression, it was plausible that induction of DR4 would be an efficient way to potentiate tumoricidal potential of TRAIL in GC. Another crucial point for increasing cell susceptibility to TRAIL is inhibition of the anti-apoptotic protein. Previous studies supported that TRAIL-resistant cells were re-sensitized by Bcl-2 or IAP antagonist $(17,18)$. Here we revealed that PTX itself markedly inhibited expression of C-IAP1, C-IAP2, Livin and Mcl-1 in both SGC-7901 and MGC-803 cells, and combined application of PTX and TRAIL exhibited similar effects. In addition, knockdown of C-IAP1 by 
siRNA augmented cytotoxic potential of TRAIL indicating PTX-mediated suppression of anti-apoptotic proteins contributed to the sensitizing effect.

Noteworthy, we found MAPKs were involved in the PTX-mediated sensitization to TRAIL. MAPK pathway, regulating cell proliferation, differentiation, mitosis and apoptosis, mainly consists of ERK, JNK1/2 and p38 MAPK members. They are frequently activated in GC (35). In this study, we found MAPKs (including ERK, JNK and p38) acted as negative regulators in TRAIL-induced apoptosis. PTX, however, suppressed MAPK activation, abolished the inhibition effect, and therefore partly restored TRAIL sensitivity. We also revealed suppression of JNK by the specific inhibitor SP600125, which showed the most effective sensitization effect. Consistent with the results of Mucha et al (19), JNK inhibition sensitised hepatocellular carcinoma cells to TRAIL. Similarly, ERK abrogated TRAIL-induced apoptosis by phosphorylating pro-caspase- 8 and inhibited the cleavage of Bid $(36,37)$. However, other groups reported that enforced activation of ERK and p38 conferred sensitization of tumor cells to TRAIL by suppressing expression of FLIP and increasing expression of DRs $(34,38)$. These inconsistent results indicate that MAPK signaling may play various roles in the way the diverse cancer cells react to TRAIL. Moreover, our results showed that MAPKs played opposing roles on modulating DRs and only JNK inhibitor could induce both DR4 and DR5 expression. In agreement with our results, Kim et al (39) have shown SP600125 upregulated DRs surface expression on hepatocellular cancer cells. We also found MAPK inhibitors could suppress expression of anti-apoptotic protein C-IAP2 and Mcl-1. All these results suggested that inhibition of MAPKs was involved in TRAIL-sensitizing effect of PTX.

In summary, the present study suggested that PTX sensitized resistant GC cells to TRAIL-mediated tumoricidal effect both in vitro and in vivo. Potentiation of mitochondrial apoptotic signals, upregulation of death receptors, downregulation of anti-apoptotic proteins and inactivation of MAPKs were all involved in the synergistic interaction. Our findings strongly suggested that the combination of PTX with TRAIL could serve as a new therapeutic strategy for GC. Further study on the potential application in this direction is warranted.

\section{Acknowledgements}

The present study was supported in part by the National Nature Science Foundation of China (nos. 81402308 and 81301874), the Natural Science Foundation of Beijing (no. 7132051), the 985 special project sponsored by the Peking University Health Science Center (no. 2013-5-09), and the Ministry of Science and Technology of the People's Republic of China (Nos. 2014AA020603, 2012AA02A203-B01, 2012AA02A504-B01, 2012AA020101 and 2014AA020603).

\section{References}

1. Torre LA, Bray F, Siegel RL, Ferlay J, Lortet-Tieulent J and Jemal A: Global cancer statistics, 2012. CA Cancer J Clin 65: 87-108, 2015

2. Call JA, Eckhardt SG and Camidge DR: Targeted manipulation of apoptosis in cancer treatment. Lancet Oncol 9: 1002-1011, 2008.
3. Pitti RM, Marsters SA, Ruppert S, Donahue CJ, Moore A and Ashkenazi A: Induction of apoptosis by Apo-2 ligand, a new member of the tumor necrosis factor cytokine family. J Biol Chem 271: 12687-12690, 1996.

4. Kischkel FC, Lawrence DA, Chuntharapai A, Schow P, Kim KJ and Ashkenazi A: Apo2L/TRAIL-dependent recruitment of endogenous FADD and caspase- 8 to death receptors 4 and 5 . Immunity 12: 611-620, 2000.

5. Samraj AK, Keil E, Ueffing N, Schulze-Osthoff K and Schmitz I: Loss of caspase-9 provides genetic evidence for the type I/ II concept of CD95-mediated apoptosis. J Biol Chem 281: 29652-29659, 2006.

6. Herbst RS, Eckhardt SG, Kurzrock R, Ebbinghaus S, O'Dwyer PJ, Gordon MS, Novotny W, Goldwasser MA, Tohnya TM, Lum BL, et al: Phase I dose-escalation study of recombinant human Apo2L/TRAIL, a dual proapoptotic receptor agonist, in patients with advanced cancer. J Clin Oncol 28: 2839-2846, 2010.

7. Soria JC, Smit E, Khayat D, Besse B, Yang X, Hsu CP, Reese D, Wiezorek $\mathrm{J}$ and Blackhall $\mathrm{F}$ : Phase $1 \mathrm{~b}$ study of dulanermin (recombinant human Apo2L/TRAIL) in combination with paclitaxel, carboplatin, and bevacizumab in patients with advanced non-squamous non-small-cell lung cancer. J Clin Oncol 28: 1527-1533, 2010.

8. Wiezorek J, Holland P and Graves J: Death receptor agonists as a targeted therapy for cancer. Clin Cancer Res 16: 1701-1708, 2010.

9. Dimberg LY, Anderson CK, Camidge R, Behbakht K, Thorburn A and Ford HL: On the TRAIL to successful cancer therapy? Predicting and counteracting resistance against TRAIL-based therapeutics. Oncogene 32: 1341-1350, 2013.

10. Prasad S, Kim JH, Gupta SC and Aggarwal BB: Targeting death receptors for TRAIL by agents designed by Mother Nature. Trends Pharmacol Sci 35: 520-536, 2014.

11. Horak P, Pils D, Haller G, Pribill I, Roessler M, Tomek S, Horvat R, Zeillinger R, Zielinski C and Krainer M: Contribution of epigenetic silencing of tumor necrosis factor-related apoptosis inducing ligand receptor 1 (DR4) to TRAIL resistance and ovarian cancer. Mol Cancer Res 3: 335-343, 2005.

12. Bin L, Thorburn J, Thomas LR, Clark PE, Humphreys R and Thorburn A: Tumor-derived mutations in the TRAIL receptor DR5 inhibit TRAIL signaling through the DR4 receptor by competing for ligand binding. J Biol Chem 282: 28189-28194, 2007.

13. Zhang Y and Zhang B: TRAIL resistance of breast cancer cells is associated with constitutive endocytosis of death receptors 4 and 5. Mol Cancer Res 6: 1861-1871, 2008.

14. Grotzer MA, Eggert A, Zuzak TJ, Janss AJ, Marwaha S, Wiewrodt BR, Ikegaki N, Brodeur GM and Phillips PC: Resistance to TRAIL-induced apoptosis in primitive neuroectodermal brain tumor cells correlates with a loss of caspase- 8 expression. Oncogene 19: 4604-4610, 2000.

15. Ricci MS, Kim SH, Ogi K, Plastaras JP, Ling J, Wang W, Jin Z, Liu YY, Dicker DT, Chiao PJ, et al: Reduction of TRAIL-induced Mcl-1 and cIAP2 by c-Myc or sorafenib sensitizes resistant human cancer cells to TRAIL-induced death. Cancer Cell 12: 66-80, 2007.

16. Munshi A, Pappas G, Honda T, McDonnell TJ, Younes A, Li Y and Meyn RE: TRAIL (APO-2L) induces apoptosis in human prostate cancer cells that is inhibitable by Bcl-2. Oncogene 20: 3757-3765, 2001

17. Finlay D, Vamos M, González-López M, Ardecky RJ, Ganji SR, Yuan H, Su Y, Cooley TR, Hauser CT, Welsh K, et al: Small-molecule IAP antagonists sensitize cancer cells to TRAIL-induced apoptosis: Roles of XIAP and cIAPs. Mol Cancer Ther 13: 5-15, 2014.

18. Kocab AJ, Veloso A, Paulsen MT, Ljungman M and Duckett CS: Effects of physiological and synthetic IAP antagonism on c-IAPdependent signaling. Oncogene 34: 5472-5481, 2015.

19. Mucha SR, Rizzani A, Gerbes AL, Camaj P, Thasler WE, Bruns CJ, Eichhorst ST, Gallmeier E, Kolligs FT, Göke B, et al: JNK inhibition sensitises hepatocellular carcinoma cells but not normal hepatocytes to the TNF-related apoptosis-inducing ligand. Gut 58: 688-698, 2009.

20. Lee HH, Jeong JW, Lee JH, Kim GY, Cheong J, Jeong YK, Yoo YH and Choi YH: Cordycepin increases sensitivity of Hep3B human hepatocellular carcinoma cells to TRAIL-mediated apoptosis by inactivating the JNK signaling pathway. Oncol Rep 30: 1257-1264, 2013. 
21. Liu J, Qu X, Xu L, Zhang Y, Qu J, Hou K and Liu Y: Phosphoinositide 3-kinase/Akt and nuclear factor $\kappa \mathrm{B}$ pathways are involved in tumor necrosis factor-related apoptosis-inducing ligand resistance in human gastric cancer cells. Mol Med Rep 3: 491-496, 2010.

22. Wan Z, Pan H, Liu S, Zhu J, Qi W, Fu K, Zhao T and Liang J: Downregulation of SNAIL sensitizes hepatocellular carcinoma cells to TRAIL-induced apoptosis by regulating the NF- $\kappa \mathrm{B}$ pathway. Oncol Rep 33: 1560-1566, 2015.

23. Nazim UM, Jeong JK, Seol JW, Hur J, Eo SK, Lee JH and Park SY: Inhibition of the autophagy flux by gingerol enhances TRAIL-induced tumor cell death. Oncol Rep 33: 2331-2336, 2015.

24. Liu YJ, Lin YC, Lee JC, Kuo SC, Ho CT, Huang LJ, Kuo DH and Way TD: CCT327 enhances TRAIL-induced apoptosis through the induction of death receptors and downregulation of cell survival proteins in TRAIL-resistant human leukemia cells. Oncol Rep 32: 1257-1264, 2014.

25. Pasquier E, Carré M, Pourroy B, Camoin L, Rebaï O, Briand C and Braguer D: Antiangiogenic activity of paclitaxel is associated with its cytostatic effect, mediated by the initiation but not completion of a mitochondrial apoptotic signaling pathway. Mol Cancer Ther 3: 1301-1310, 2004.

26. Gong J, Yang D, Kohanim S, Humphreys R, Broemeling L and Kurzrock R: Novel in vivo imaging shows up-regulation of death receptors by paclitaxel and correlates with enhanced antitumor effects of receptor agonist antibodies. Mol Cancer Ther 5: 2991-3000, 2006.

27. Hunter TB, Manimala NJ, Luddy KA, Catlin T and Antonia SJ: Paclitaxel and TRAIL synergize to kill paclitaxel-resistant small cell lung cancer cells through a caspase-independent mechanism mediated through AIF. Anticancer Res 31: 3193-3204, 2011.

28. Nimmanapalli R, Perkins CL, Orlando M, O'Bryan E, Nguyen D and Bhalla KN: Pretreatment with paclitaxel enhances apo-2 ligand/tumor necrosis factor-related apoptosis-inducing ligandinduced apoptosis of prostate cancer cells by inducing death receptors 4 and 5 protein levels. Cancer Res 61: 759-763, 2001.

29. Lee MJ, Ye AS, Gardino AK, Heijink AM, Sorger PK, MacBeath G and Yaffe MB: Sequential application of anticancer drugs enhances cell death by rewiring apoptotic signaling networks. Cell 149: 780-794, 2012

30. Tsuburaya A, Yoshida K, Kobayashi M, Yoshino S, Takahashi M, Takiguchi N, Tanabe K, Takahashi N, Imamura H, Tatsumoto N, et al: Sequential paclitaxel followed by tegafur and uracil (UFT) or S-1 versus UFT or S-1 monotherapy as adjuvant chemotherapy for T4a/b gastric cancer (SAMIT): A phase 3 factorial randomised controlled trial. Lancet Oncol 15: 886-893, 2014.
31. Trivedi R, Maurya R and Mishra DP: Medicarpin, a legume phytoalexin sensitizes myeloid leukemia cells to TRAIL-induced apoptosis through the induction of DR5 and activation of the ROS-JNK-CHOP pathway. Cell Death Dis 5: e1465, 2014.

32. McDaid HM and Horwitz SB: Selective potentiation of paclitaxel (taxol)-induced cell death by mitogen-activated protein kinase kinase inhibition in human cancer cell lines. Mol Pharmacol 60: 290-301, 2001

33. von Haefen C, Wieder T, Essmann F, Schulze-Osthoff K, Dörken B and Daniel PT: Paclitaxel-induced apoptosis in BJAB cells proceeds via a death receptor-independent, caspases3/-8-driven mitochondrial amplification loop. Oncogene 22: 2236-2247, 2003.

34. Do MT, Na M, Kim HG, Khanal T, Choi JH, Jin SW, Oh SH, Hwang IH, Chung YC, Kim HS, et al: Ilimaquinone induces death receptor expression and sensitizes human colon cancer cells to TRAIL-induced apoptosis through activation of ROS-ERK/ p38 MAPK-CHOP signaling pathways. Food Chem Toxicol 71: $51-59,2014$

35. Paterson AL, Shannon NB, Lao-Sirieix P, Ong CA, Peters CJ, O'Donovan $\mathrm{M}$ and Fitzgerald RC: A systematic approach to therapeutic target selection in oesophago-gastric cancer. Gut 62 : 1415-1424, 2013.

36. Söderström TS, Poukkula M, Holmström TH, Heiskanen KM and Eriksson JE: Mitogen-activated protein kinase/extracellular signal-regulated kinase signaling in activated $\mathrm{T}$ cells abrogates TRAIL-induced apoptosis upstream of the mitochondrial amplification loop and caspase-8. J Immunol 169: 2851-2860, 2002.

37. Mandal R, Raab M, Matthess Y, Becker S, Knecht R and Strebhardt K: pERK 1/2 inhibit caspase-8 induced apoptosis in cancer cells by phosphorylating it in a cell cycle specific manner. Mol Oncol 8: 232-249, 2014.

38. Yerbes R, López-Rivas A, Reginato MJ and Palacios C: Control of FLIP(L) expression and TRAIL resistance by the extracellular signal-regulated kinase1/2 pathway in breast epithelial cells. Cell Death Differ 19: 1908-1916, 2012.

39. Kim EY, Ryu JH and Kim AK: CAPE promotes TRAIL-induced apoptosis through the upregulation of TRAIL receptors via activation of p38 and suppression of JNK in SK-Hep1 hepatocellular carcinoma cells. Int J Oncol 43: 1291-1300, 2013. 